\title{
FORUM payload study for phase A/ B1 by a consortium led by OHB System AG and TAS-UK
}

S. Fray, M. Lippa, E. Semmler, W. Posselt, Ph. Giaccari, et al.

S. Fray, M. Lippa, E. Semmler, W. Posselt, Ph. Giaccari, C. A. Mastrandrea, Uwe D. Zeitner, Th. Fluegel-Paul, S. Sadlowski, Th. Kaesebier, Ch. Monte, M. Kehrt, "FORUM payload study for phase A/B1 by a consortium led by OHB System AG and TAS-UK," Proc. SPIE 11852, International Conference on Space Optics - ICSO 2020, 1185225 (11 June 2021); doi:

$10.1117 / 12.2599356$

SPIE Event: International Conference on Space Optics - ICSO 2021, 2021, Online Only 


\section{International Conference on Space Optics-ICSO 2020}

Virtual Conference

30 March-2 April 2021

Edited by Bruno Cugny, Zoran Sodnik, and Nikos Karafolas
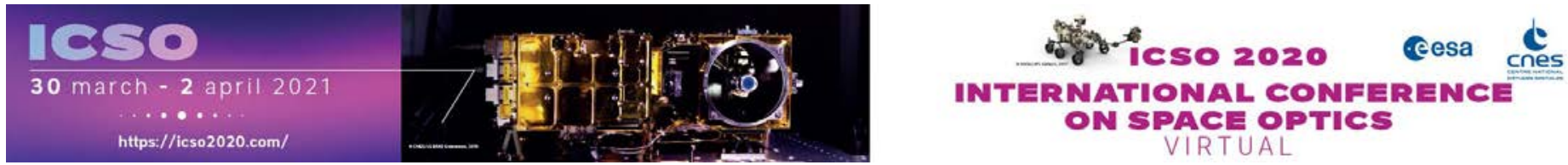

FORUM payload study for phase $A / B 1$ by a consortium led by OHB System AG and TAS-UK

\section{Cesa issopocestings denes}




\title{
FORUM payload study for phase A/B1 by a consortium led by OHB System AG and TAS-UK
}

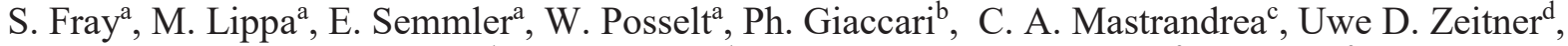 \\ Th. Fluegel-Paul ${ }^{d}$, S. Sadlowski ${ }^{d}$, Th. Kaesebiere, Ch. Monte ${ }^{\mathrm{f}}$, M. Kehrt ${ }^{\mathrm{f}}$ \\ ${ }^{a}$ OHB System AG, Manfred-Fuchs-Straße 1, D-82234 Wessling, Germany; \\ ${ }^{b}$ Micos Engineering GmbH, Ueberlandstrasse 129, CH-8600 Duebendorf, Switzerland; \\ ${ }^{\mathrm{c}}$ Leonardo S.p.A, Via A. Einstein 35, Campi Bisenzio (FI) - 50013, Italy; \\ ${ }^{\mathrm{d}}$ Fraunhofer-Institut für Angewandte Optik und Feinmechanik IOF, \\ Albert-Einstein-Str. 7, D-07745 Jena, Germany; \\ ${ }^{\mathrm{e}}$ Institut für Angewandte Physik Mikrostrukturierung und Mikrooptik, Friedrich-Schiller-Universität \\ Jena, Albert-Einstein-Str.15 D-07745 Jena, Germany \\ fPhysikalisch-Technische Bundesanstalt, Abbestrasse 2-12, D-10587 Berlin, Germany
}

\begin{abstract}
In 2019, the FORUM mission (Far-infrared Outgoing Radiation Understanding and Monitoring) has been selected by ESA as Earth Explorer 9 mission. FORUM aims to record the emission spectrum of the Earth's stratosphere and troposphere in the spectral range of 100 to $1600 \mathrm{~cm}^{-1}$ (i.e. $6.25 \mu \mathrm{m}$ to $100 \mu \mathrm{m}$ ). It will measure the spectral features of the far-infrared contribution to the Earth radiation budget with the focus on the water vapour contribution, the cirrus cloud properties, and the ice/snow surface emissivity. FORUM's primary optical instrument is a non-imaging Fourier Transform Spectrometer (FTS) scanning the Earth atmosphere in a step-and-stare acquisition mode sounding a $15 \mathrm{~km}$ ground sampling with a ground sampling distance of $100 \mathrm{~km}$. A consortium led by TAS-UK as mission and platform prime and OHB as instrument prime are currently developing the satellite and payload together with Leonardo Italy and MICOS during an ESA phase A / B1 study. This consortium is one of the 2 independent consortia selected for FORUM's phase A/B1.

The paper will describe the design concept of the FORUM FTS instrument with main focus on the optical design. The key optical subsystems are the pointing unit and a double pendulum interferometer with an entrance three-mirror-anastigmatic telescope. The prediction of the performance with respect to threshold and goal requirements will be presented. Furthermore, the status of the beam splitter pre-development will be reported, in particular the development of an optical element based on a CVD-diamond substrate and a technology to manufacture broad band anti-reflective microstructures on a diamond surface. The microstructures are designed to suppress parasitic reflections in the spectral range between $6.25 \mu \mathrm{m}$ and $100 \mu \mathrm{m}$. The highest efficiency occurs between 13 and $30 \mu \mathrm{m}$, where the Fresnel reflection is reduced from $17 \%$ down to below $3.5 \%$.
\end{abstract}

\section{INTRODUCTION}

ESA has selected the FORUM mission (Far-infrared Outgoing Radiation Understanding and Monitoring) for the ninth Earth Explorer mission within ESA's Earth Observation Program. The goal of the FORUM mission is to measure thermal radiation losses of the Earth atmosphere, in particular addressing the water vapour contribution related to the continuum absorption in the rotational band, the cirrus cloud properties, and the ice/snow surface emissivity. FORUM will be the first satellite mission which provides spectrally-resolved measurements in the far-infrared range, and this way helps to improve the accuracy of future climate change predictions.

FORUM will record detailed far infrared spectral features emitted by the top-of-atmosphere in the spectral range from 100 to $1600 \mathrm{~cm}^{-1}(100 \mu \mathrm{m}$ to $6.25 \mu \mathrm{m})$. FORUM sounds a circular area of $15 \mathrm{~km}$ in diameter on Earth nadir-looking in a continuous step-and-stare mode from an altitude between $817 \mathrm{~km}$ and $847 \mathrm{~km}$. FORUM payload contains two instruments. The primary one is the FSI, a single-pixel Fourier Transform Spectrometer with a spectral resolution better than $0.5 \mathrm{~cm}^{-1}$. The FSI ground sample is co-located with the footprint of the FEI, the FORUM Embedded Imager. The FEI is a thermalinfrared-imager used to determine scene heterogeneity. Its spectral channel is centered at $10.5 \mu \mathrm{m}$ with a bandwidth of 1.5 $\mu \mathrm{m}$. 
A consortium led by TAS-UK as mission and platform prime and OHB as instrument prime develops the satellite and payload together with Leonardo Italy and MICOS Engineering during an ESA paid phase A / B1 study. This consortium is one of the 2 independent consortia selected for FORUM's phase A/B1. The presented instrument design represents the status at the end of phase B1.

\section{KEY REQUIREMENTS}

The design-relevant mission requirements are given in Table 1.

Table 1: Design-relevant mission requirements

\begin{tabular}{|c|c|c|}
\hline Parameter & Threshold & Goal \\
\hline Altitude [km] & $817-847$ & \\
\hline $\begin{array}{l}\text { Spatial Sampling Distance } \\
{[\text { [km] }}\end{array}$ & 100 & 70 \\
\hline $\begin{array}{c}\text { System Integrated Energy } \\
\text { (SIE) }\end{array}$ & $>91 \%$ over $15 \mathrm{~km}$ circle & \\
\hline Wavenumber $\left[\mathrm{cm}^{-1}\right]$ & {$[100 ; 1600]$} & \\
\hline Spectral resolution $\left[\mathrm{cm}^{-1}\right]$ & $<0.5$ & $<0.36$ \\
\hline NESR $\left[\mathrm{mW} /\left(\mathrm{m}^{2} \mathbf{s r} \mathrm{cm}^{-1}\right)\right]$ & $\begin{array}{c}0.6 \text { inside }[200 ; 800 \mathrm{~cm}-1] \\
2.0 \text { else }\end{array}$ & $\begin{array}{c}0.4 \text { inside }[200 ; 800 \mathrm{~cm}-1] \\
1.0 \mathrm{else}\end{array}$ \\
\hline
\end{tabular}

The orbit altitude drives the instrument dimensions, keeping the system f-number constant. The SSD drives the interferogram acquisition time. The SIE drives the effective focal length. The wavenumber range is a critical parameter for selection of materials (e.g. diamond) and technologies (e.g. pyro-electric detector). The spectral resolution drives the NESR linearly. The NESR drives the aperture (throughput), efficiency and tolerable detector noise power.

The optical design parameters are:

Table 2: Optical design parameters

\begin{tabular}{|l|c|}
\hline \multicolumn{1}{|c|}{ Parameter } & Value \\
\hline Pixel size $[\mathbf{m m}]$ & 2, square \\
\hline Entrance aperture $[\mathbf{m m}]$ & 80 \\
\hline Telescope magnification & 2.4 \\
\hline
\end{tabular}

The FSI design is compatible with the goal NESR and the threshold SSD and threshold spectral resolution. Goal spectral resolution is possible for infrequent in-orbit calibration.

\section{INSTRUMENT ARCHITECTURE}

The FORUM payload accommodates two instruments: the FORUM Sounding Instrument (FSI) and the FORUM Embedded Imager (FEI), which use the same front-end. The payload is composed of two modules:

- Instrument Optical Module (IOM), mounted on a side panel of the satellite and thermally de-coupled from it,

- Instrument Electronic Module (IEM) that is placed inside the satellite body. 
The IOM contains all optics, mechanisms, calibration blackbody unit (BBU) and near-by electronics, in particular frontend electronics including A/D conversion for all data channels (FSI detectors, laser receivers, and FEI microbolometer), the laser source with its driver, and the interface to the IEM.

Only the IEM has electrical interfaces with the spacecraft bus for power, data and housekeeping (HK) relevant to the whole instrument. The IEM contains the instrument control and data handling unit (ICU), the power supply units (PDCU) for all instrument subsystems, the mechanisms drive and control electronics and the interfacing to the bus. It is connected to the IOM by means of dedicated harness. The general functional block diagram is shown below.

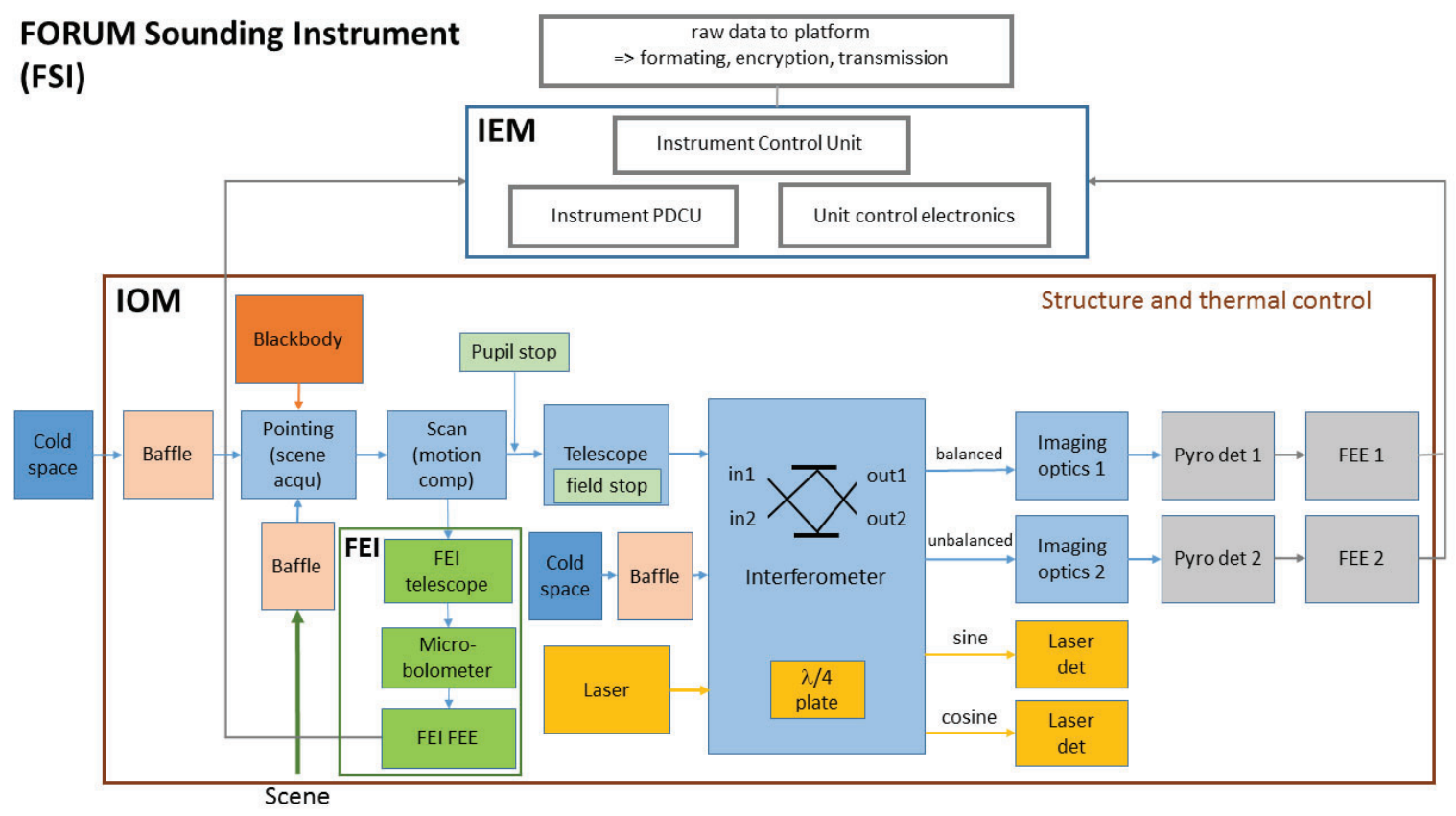

Figure 1 FORUM Functional Block Diagram

In Figure 2 the mechanical design of the instrument is illustrated. To efficiently accommodate all functional assemblies of the IOM the optical bench is populated on both sides. The entrance and cold space baffle, black body and the pointing assembly are located on the top side, while the scan assembly, the front telescope, the interferometer assembly and back telescope \& detection assembly are placed on the bottom side of the optical bench. Optically both sides are connected by an opening in the bench between the pointing and scan mirror.

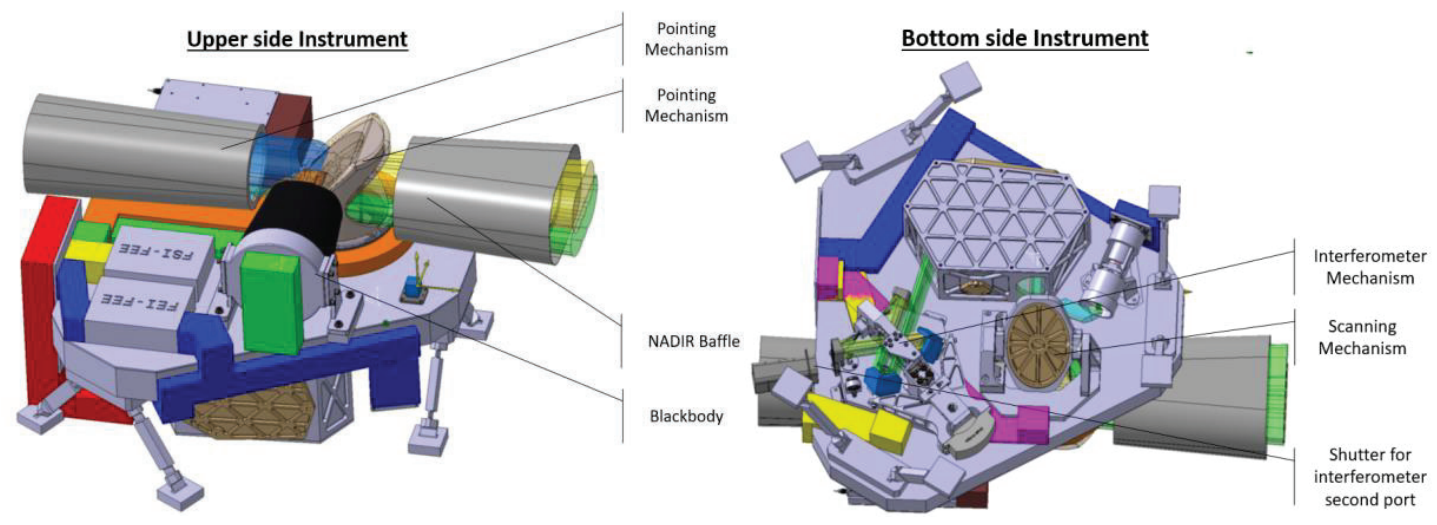

Figure 2 FORUM IOM accommodation, top view (left) and bottom view (right). 


\subsection{FSI Design Overview}

The Forum Interferometer is based on a double pendulum interferometer as a core component. In front there are located a pointing and a scan unit as well as a front telescope, which direct the light from the scene to the interferometer assembly. The detection is implemented by means of two pyro-electric detectors. The main optical element of the double pendulum is the beam splitter. It consists of two CVD diamond windows with a beam splitter coating made of germanium. The backside of the diamond window is coated with microstructures, which serves as a broadband antireflective coating. All mirrors besides the corner cubes are coated with a protected gold coating.

The functions of pointing and scanning mirrors are separated from each other and implemented in two separate functional units. This allows to optimize both units independently without compromising the development goals. For example, the scan unit needs a high angular accuracy, while the pointing mechanism requires a large angular travel range.

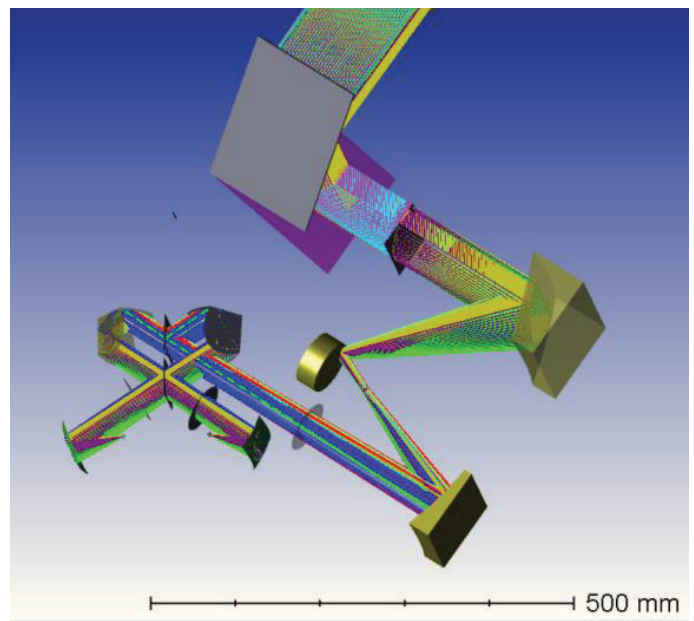

Figure 3 Optical layout of the FSI

\subsection{Front-End Functional Units}

$\underline{\text { Baffles }}$

First element in the instrument functional block diagram is the radiance source: either external sources like the scene or cold space or the internal blackbody. Baffles will reduce the stray light sensitivity, especially in orbit positions with close angular distance of the sun. Baffles are foreseen for the Nadir view and the two cold-space views.

\section{Calibration Blackbody}

The internal blackbody serves as absolute reference. Its spectral exitance is precisely known from the knowledge of the surface spectral emissivity and the knowledge of its temperature.

The blackbody shall be aperture filling. In view of the large aperture required, a grooved planar surface with a cylindrical baffle is selected as baseline. The structured surface generates multiple reflections and boosts the emissivity. The structure of the pointing mirror shuts the blackbody when not operational and keep the blackbody at equilibrium conditions.

The blackbody will be operated floating at the temperature of its environment. This approach has the advantage that nonperfect emissivity has little influence.

The FORUM Blackbody has a simple structure whose main sub-assemblies are:

- $\quad$ The V-grooved emissive plate, integrating the temperature sensors, and a baffle

- $\quad$ A housing supporting the emissive plate through thermally insulating spacers

- Two mounting brackets, to hold the blackbody through the housing. 

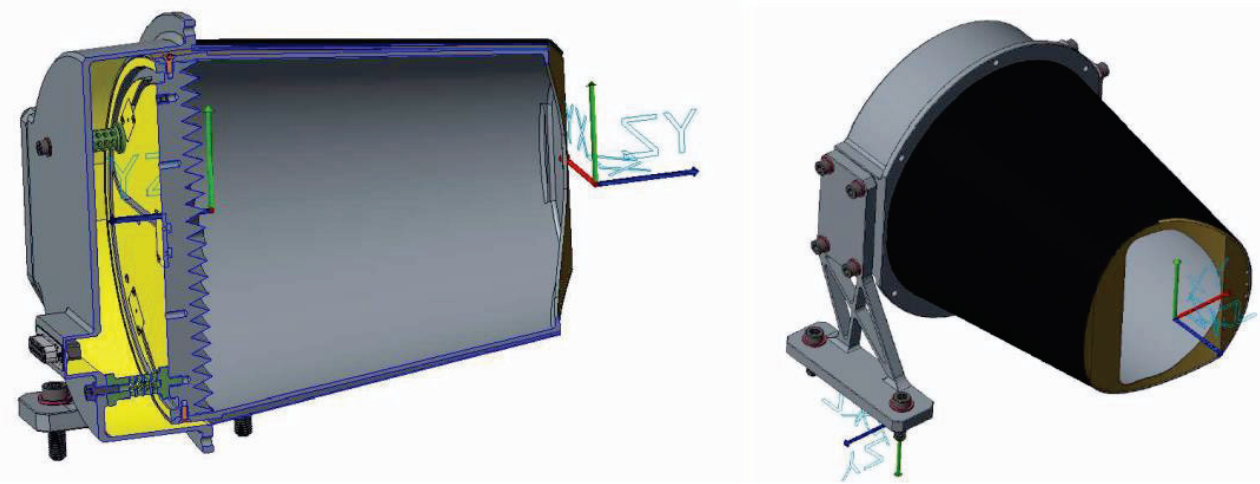

Figure 4 Calibration Blackbody.

Pointing Unit and Scan Unit

In Figure 5 and Figure 6 the operation principles and the mechanical configuration of the pointing and scan mechanisms are shown. The pointing unit is acting in across track and will be used to access Nadir direction, cold space and the internal blackbody. The pointing mirror will be rotated along an axis along the instrument LOS. This way the angle of incidence is constant and the sensitivity to polarization is minimum.

The scan unit follows the pointing unit and is acting in along track. Its task is to compensate the orbital motion and keep the LOS intersection with the Earth surface stable during the interferogram acquisition. The rotation axis is in the mirror plane.

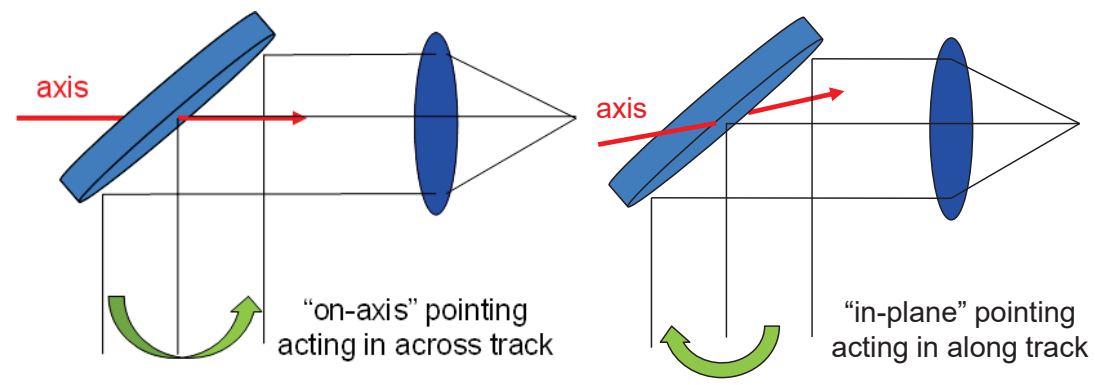

Figure 5 Operation principle of the pointing mirror (left) and scan mirror (right)
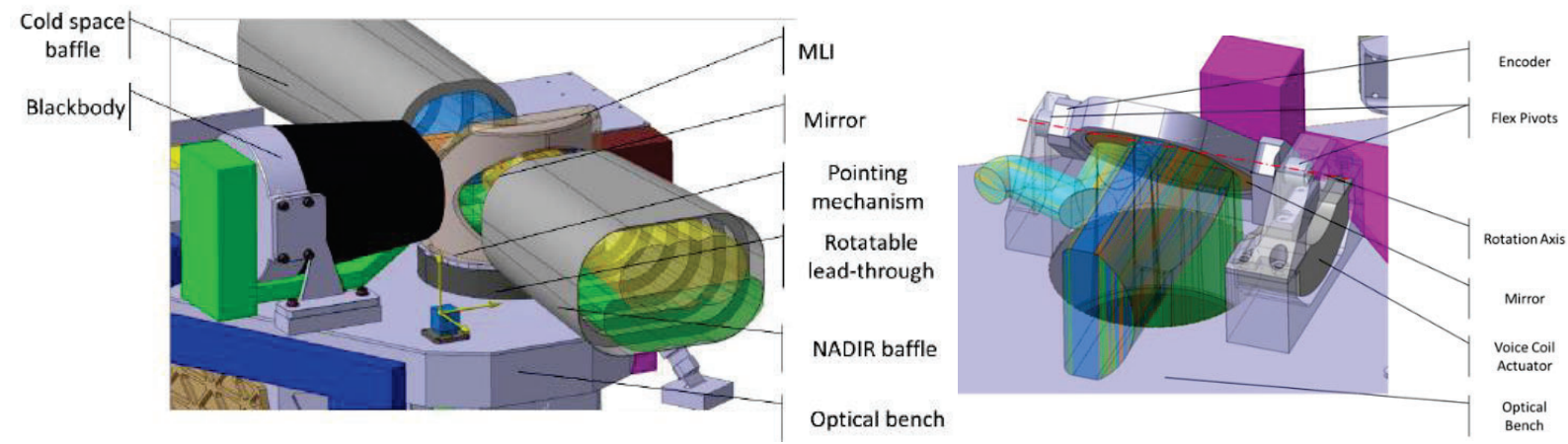

Figure 6 Mechanical configuration of pointing (left) and scan mechanism (right) 


\subsection{Additional FSI Functional Units}

\section{Telescope}

The telescope is an off-axis three-mirror afocal with a magnification of 2.4. The selection of the pupil magnification factor is a trade-off between the available corner cube size and acceptable self-apodisation. The primary and tertiary mirrors have parabolic concave surface form while the secondary is an aspheric convex mirror. The telescope reduces the beam diameter inside the interferometer, in order to minimize the dimensions of the beam splitter and corner cubes.

Another task is to provide an accessible intermediate focal plane, where the instrument field stop will be placed. It defines a circular field of view of $+/-0.507^{\circ}$. The telescope entrance pupil is located in front of the first mirror and allows for placing a pupil stop at this position. The F/number at the intermediate focal plane is 4.23 .

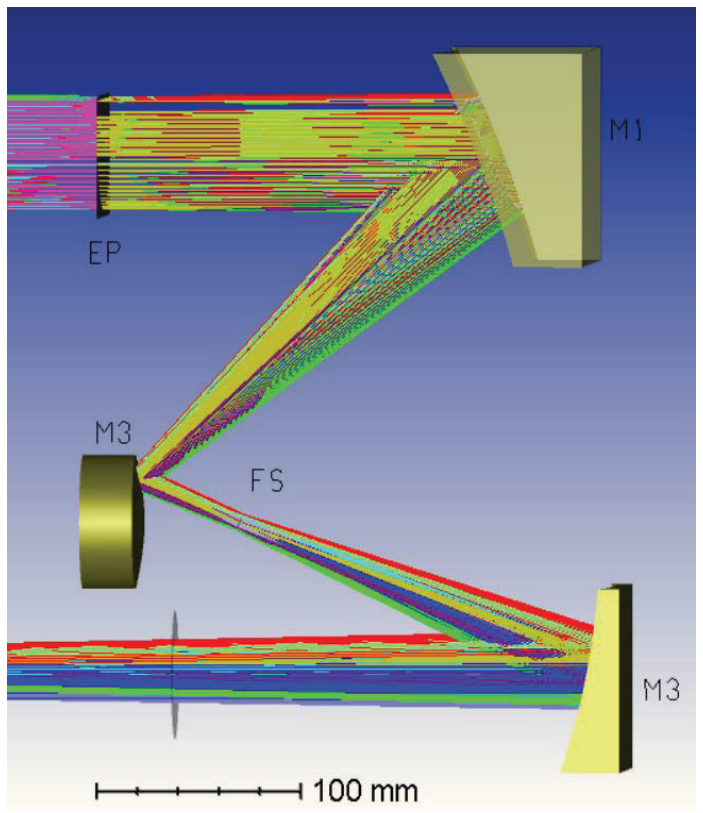

Figure 7 Three mirror afocal front telescope

\section{Pupil Stop and Field Stop}

The pupil stop is located in the plane of the telescope entrance pupil and defines the instrument entrance aperture. The image of the pupil stop is in the symmetry plane of the corner cubes (symmetric to the two reflections inside the corner cube). It is D-shaped in order to minimize the dimensions of the corner cubes. Large corner cubes are not only driving the instrument dimensions and mass, but also the criticality and costs of the corner cubes.

Radiometrically a round shaped pupil size of $80 \mathrm{~mm}$ in diameter is required. The adequate D-shaped pupil with a corresponding area is depicted in Figure 8, left side. Its area fits into a rectangle with dimensions of $110.06 \mathrm{~mm} \times 53.67$ $\mathrm{mm}$. On the right side of Figure 8 it is illustrated how the D-shaped incoming and reflected beam fits into the clear aperture of the corner cube, which is $71 \mathrm{~mm}$ in diameter. 

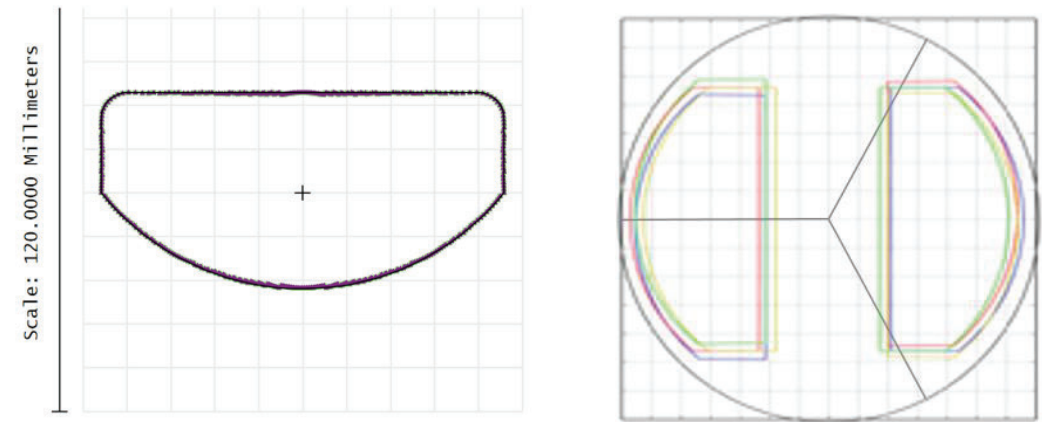

Figure 8 D-shaped entrance pupil (left) and incoming and reflected beam footprints on the corner cube's clear aperture (right).

The field stop is located in the intermediate focal plane of the telescope and is the limiting stop defining the field of the instrument.

Thus, the first two mirrors of the telescope define the geometrical performance of the instrument. All optical elements following these two are oversized with reduced requirements on image quality. Especially the oversized detector will then only serve as "photon bucket" and can have a square shape, which provides better uniformity. The oversizing of optical elements is uncritical, because the level of in-field stray light is proportional to the nominal signal, so it can be removed by calibration.

\section{Interferometer}

The interferometer generates interferograms, which are the Fourier transform of the spectrum of the recorded radiation. The original spectrum can be reconstructed from the recorded interferogram by the inverse Fourier transform.

The dual port double pendulum concept was selected as baseline and is a mixture of Michelson and Mach-Zehnder. It benefits from the compact and simple design of a Michelson interferometer and from separated and accessible 4 beam splitter ports of a Mach-Zehnder design. Comparing with other potential candidates it is the most compact and simple setup, promising high performance. In addition, the principle double pendulum concept has flight heritage. In Figure 9 the optical design of the interferometer is depicted, and in Table 3 its key parameter are listed.

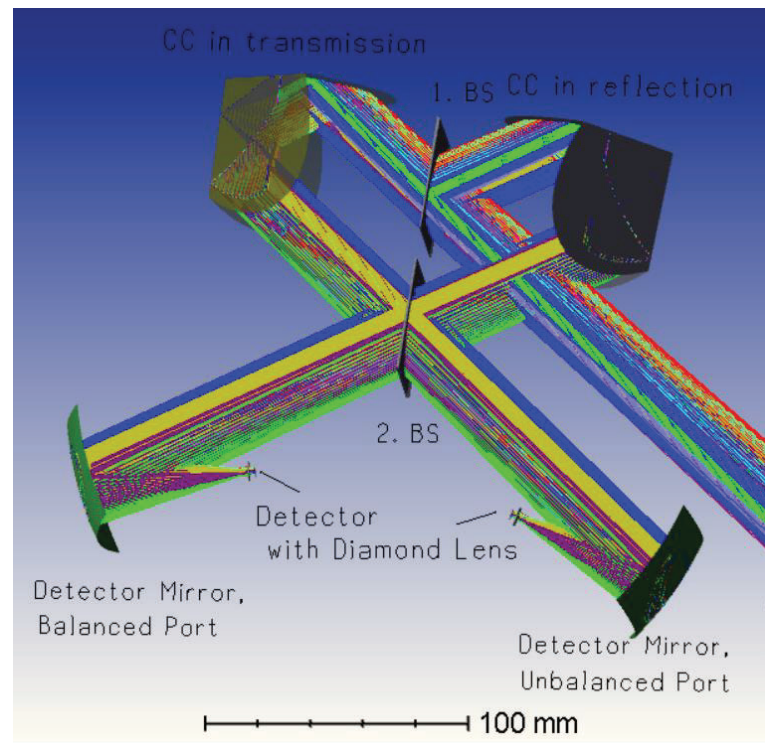

Figure 9 Optical design of the interferometer assembly 
Table 3 Interferometer parameter

\begin{tabular}{|l|l|}
\hline Parameters & Value \\
\hline $\begin{array}{l}\text { Interferometer entrance pupil } \\
\text { equivalent diameter }\end{array}$ & $33.3 \mathrm{~mm}$ \\
\hline Beam divergence & $+/-1.217^{\circ}$ \\
\hline MOPD & $13.5 \mathrm{~mm}$ \\
\hline Spectral resolution & $0.48 \mathrm{~cm}-1$ at $1600 \mathrm{~cm}^{-1}$ \\
\hline Beam splitter & Wedged diamond plates \\
\hline Cube corners & $\begin{array}{l}\text { Monolithic, bare gold coated } \\
71 \text { mm (clear aperture diameter) }\end{array}$ \\
\hline
\end{tabular}

\section{Interferometer Optics}

The interferometer is a dual-port design, where both input and output ports are accessible. The optics consists of a beamsplitter unit, which carries the beam splitter and the beam recombiner. The beam splitter and recombiner substrates will be thin, wedged plates made of CVD diamond (center thickness $<1 \mathrm{~mm}$ ). The beam-splitting capability is realized by a Germanium coating on these substrates. The transmission performance of the diamond beam splitter is enhanced by an anti-reflective (AR) structure. In Figure 10 the bare diamond substrate and the Ge coated diamond window are shown. Both coating developments were conducted under the lead of the Fraunhofer-Institut für Angewandte Optik und Feinmechanik IOF in Jena, Germany. In Figure 11 on the left a scanning electron microscope picture depicts the detailed topology of the microstructures. On the right side a measurement of the transmission through the microstructured diamond surface is shown. The measurements were performed at the German national institute of standards Physikalisch-Technische Bundesanstalt (PTB) in Berlin, Germany over the entire spectral range from $6.25 \mu \mathrm{m}$ till $100 \mu \mathrm{m}$. Diamond exhibits a large refractive index of 2.4 over the target spectral range, which leads to large Fresnel losses of about $17 \%$ or a transmission reduction to $83 \%$. Application of microstructures yields a significant increase of transmission. The highest AR efficiency occurs between 13 and $30 \mu \mathrm{m}$, where the reflection is reduced from $17 \%$ down to below $3.5 \%$.
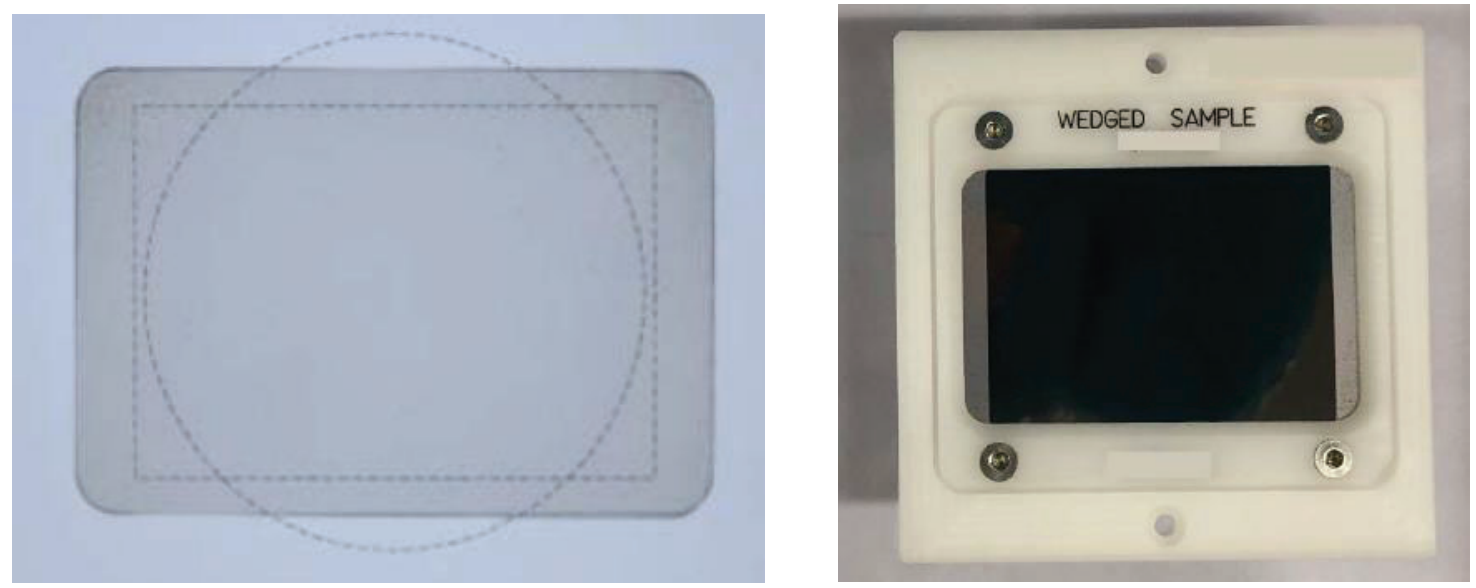

Figure 10 Uncoated (left) and Ge coated (right) wedged diamond beam splitter 

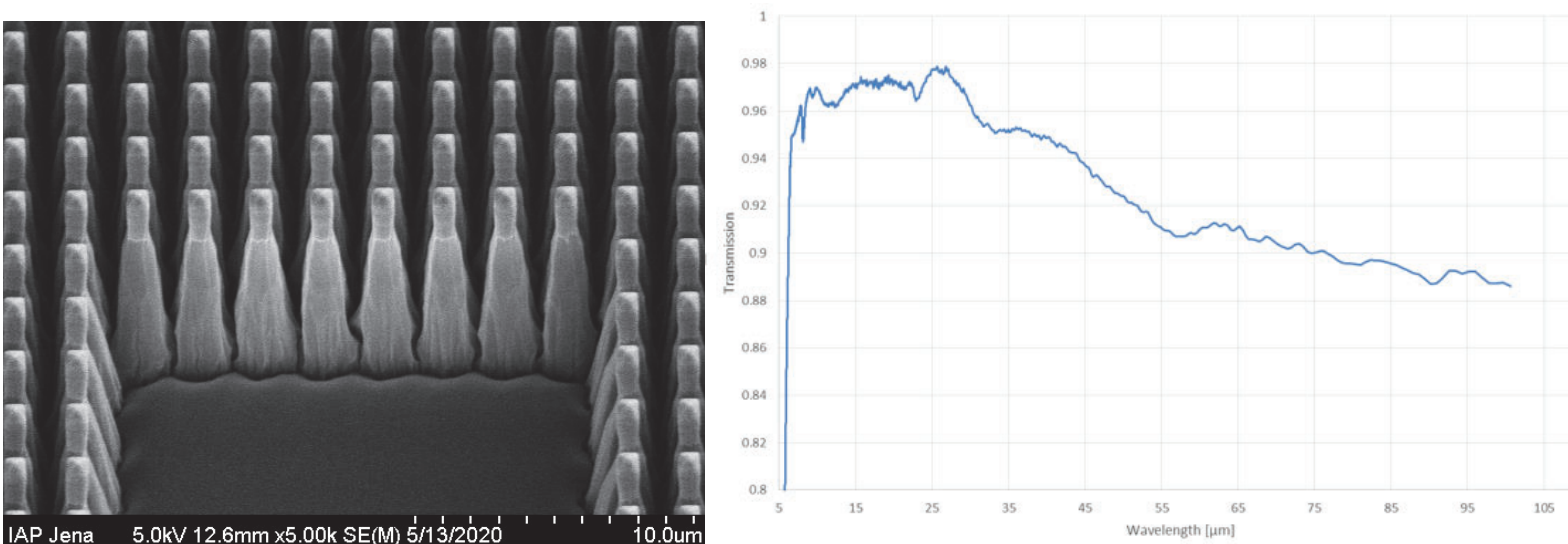

Figure 11 Diamond AR structure (left) and transmission (right) measurement of the microstructured surface

The angle of incidence on the beam splitter is $33^{\circ}$. It deviates from the conventional configuration with $45^{\circ}$ as commonly used in Michelson Interferometers. The value represents a trade-off between a low polarisation sensitivity, a short pendulum arm length and sufficient mechanical clearance between the incoming light beam and the corner cubes.

The retro-reflectors are monolithic aluminum corner cubes, with gold coating. Inside the cubes the D-shaped input beams will be laterally shifted, such that the output beams will not overlap the input beams. The shape of the input beam leaves enough space for the redundant reference laser beams and an additional window for alignment in the visible.

$\underline{\text { Interferometer Mechanism }}$

The drive unit is a double pendulum arrangement with a central bearing and two arms carrying the corner cubes. Rotation of the central bearing results in an anti-phase displacement of the two corner cubes, thereby doubling the phase shift between the two beams.

The interferometer structure carries the beam splitter unit, the double pendulum drive and elements of the Metrology System.

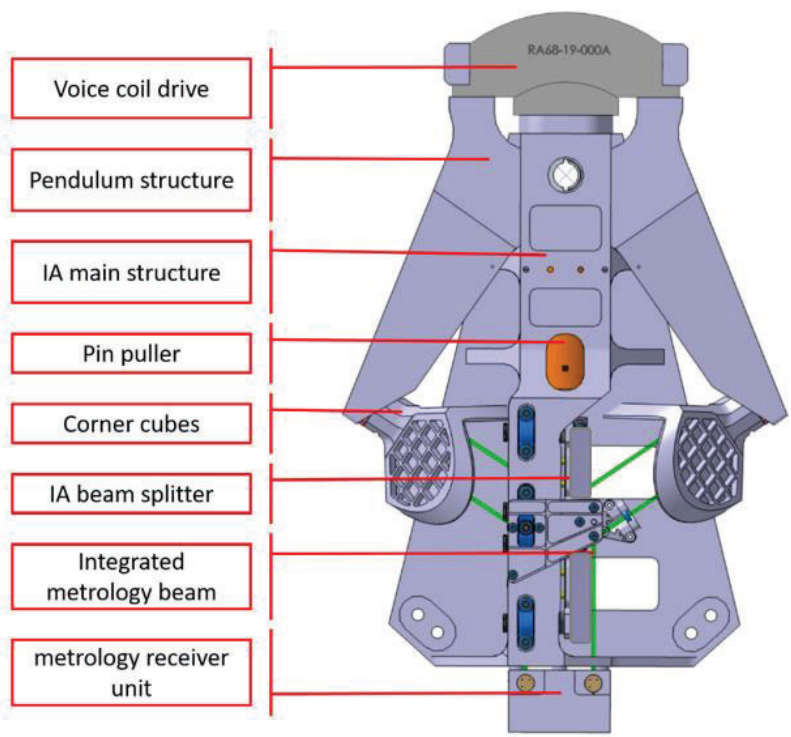

Figure 12 Interferometer Assembly 


\section{Beam Splitter Assembly}

The beam splitter assembly is the core element of the Interferometer Assembly (IA). It consists of two separate CVD diamond beam splitter windows that can be independently aligned via two separate beam splitter mounts.

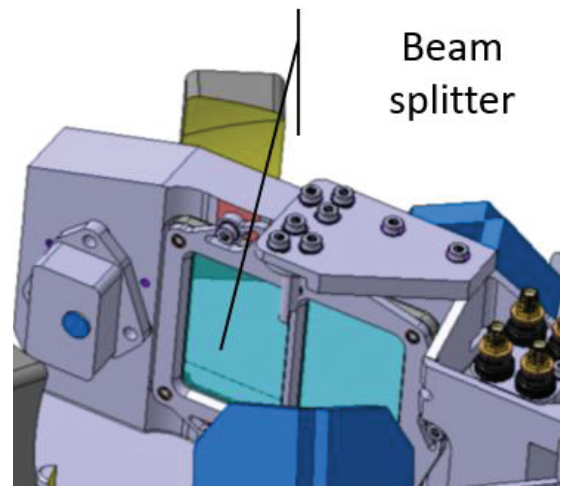

Figure 13 Beam splitter assemblies mounted in interferometer assembly structure (phase A)

The beam splitter mounts are connected to the structure with a bipod system. During the IA integration procedure beam splitter windows are pre-integrated into their mounts, which are already equipped with their bipod system that connects them to the main structure.

These Beam Splitter assemblies are then placed into the interferometer. During this step the in plane bipod position is fixed with respect to the IA main structure, while the position of the out of plane bipod can still be moved to allow for the required alignment of the beam splitter windows.

The main advantages of the bipod system are:

- The beam splitter system is very robust against manufacturing tolerances as the bipods filter out the machining tolerances.

- The system can be aligned without exchanging elements like shims in the process, therefore "live" alignment is possible.

- The alignment accuracy is not limited by manufacturing accuracies (e.g. shims).

- The optical performance of the beam splitter window is not impacted by alignment.

\section{Metrology System}

The metrology system will use the optics of the Interferometer. The laser beam with the wavelength of $1550 \mathrm{~nm}$ is fed into the optical path via a collimator and small folding mirrors.

The laser radiation injected to the interferometer is $45^{\circ}$ linearly polarised. The polarization allows to retrieve a sine and cosine components of the superimposed beams, and thus to determine the direction of the retro-reflector motion. This direction information is important as the interference fringes of the laser interferograms provide an absolute position reference that must be accurately maintained between two gain calibration sequences.

The redundant laser unit will be a temperature-stabilized diode laser as the simplest design solution. No absolute frequency stabilization will be implemented, as the spectral scale can easily be calibrated by known emission features of the atmosphere. Polarization-maintaining optical fibres are used to get the laser radiance into the interferometer.

The redundant laser-receiver unit measures the two polarization states and provides the sine and cosine information as generated by a quarter-wave plate in one of the two beams. The signals are converted to digital information and included in the science-data stream for data processing on-ground.

It is possible to use the signals of the metrology interferometer for closed-loop control of the interferometer drive. The trajectory drive of the pendulum interferometer is based on the absolute position. This position is tracked by a fringe counter which reads the metrology signals. 
Back Telescope \& Detection Assembly

The two Back Telescope \& Detection Assemblies receive the science signals from the Interferometer. Each assembly consists of imaging optics and the detection unit based on pyroelectric detectors.

Each imaging optics consists of a single mirror plus a diamond lens in the detector package for pupil imaging. In order to accommodate the D-shaped pupil the focusing mirrors is designed to have an aspherical surface form. The lens exhibits a plano- convex shape and is integrated in front of the detector.

Each of the two detection units consists of

- the pyroelectric detector, $\left(2 \times 2 \mathrm{~mm}, 45^{\circ}\right.$ rotated $)$

- the housing including a diamond lens,

- $\quad$ and the front-end electronics.

The pyroelectric detector has a squared sensitive areas. It is $2 \times 2 \mathrm{~mm}$ in size and will be operated at a controlled temperature around ambient $(295 \mathrm{~K} \pm 5 \mathrm{~K})$ by controlling the temperature of the detector housing. The detector will be integrated in a hermetically sealed TO5 package and includes the first amplification stage, a high ohm resistor and a JFET. The window of the package will be made from CVD diamond. Related performance losses can be accepted.

The front-end electronics will be located close to the detector and will amplify and digitize the signal. The digitized raw signals are formatted in the ICU and handed over to the platform for transmission to ground.
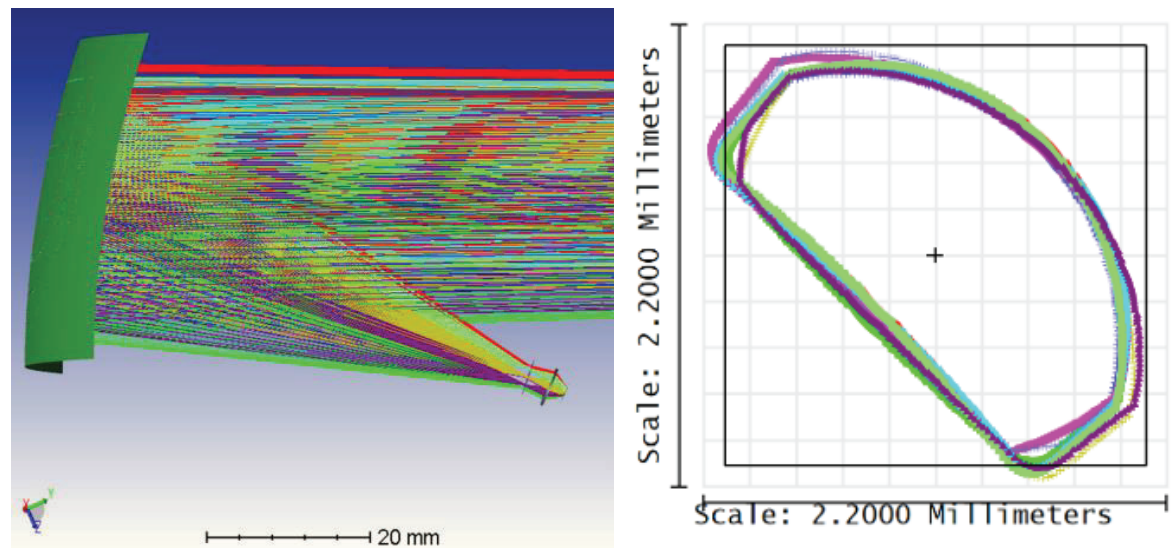

Figure 14 Optical design for the back telescope (left) and the exit pupil accommodated on the focal plane

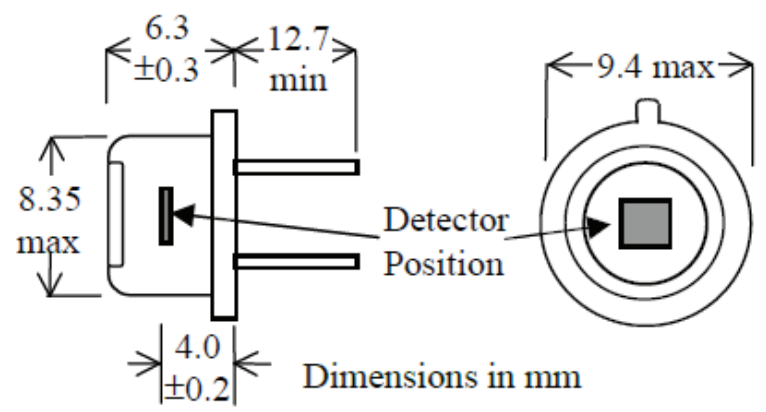

Figure 15 Pyroelectrical detector (DLATGS), Leonardo, series 106 (datasheet) 


\subsection{FEI Design}

The embedded imager is a camera operating in the thermal infrared spectral range defined by the band pass filter which has a central wavelength of $10.5 \mu \mathrm{m}$ and a FWHM of $1.5 \mu \mathrm{m}$. The FEI imager is co-aligned with the FTS interferometer and share the same pointing and scanner mechanism. In Figure 16 the FEI mechanical design is shown.

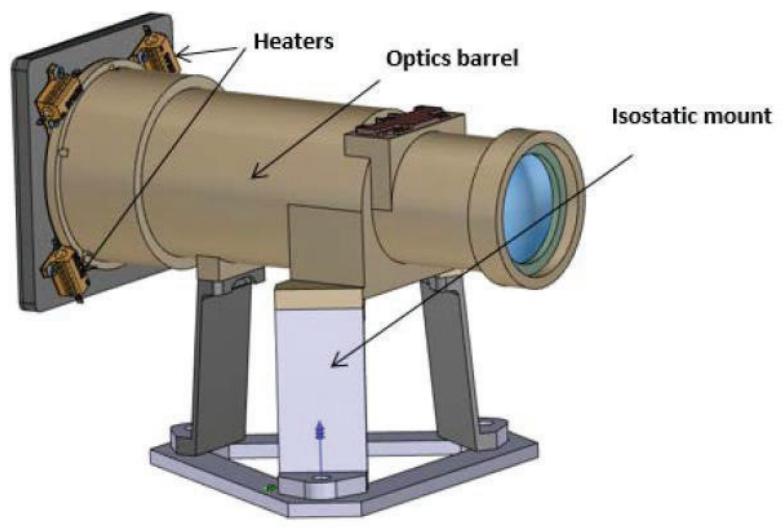

Figure 16 FEI mechanical design

The telescope for the FEI will be a refractive lens system. The focal length and thus F/\# is defined by the effective pixel size of the micro-bolometer. Several pixels of the micro-bolometer array are combined to increase the focal length. This eases the optics design and improves the performance. The first order optical parameters of the FEI in the baseline configuration are summarized in Table 4.

Table 4: First order parameters of the FEI

\begin{tabular}{|l|l|}
\hline \multicolumn{1}{|c|}{ Parameter } & \multicolumn{1}{c|}{ Refractive Design } \\
\hline Effective focal length & $49.6 \mathrm{~mm}$ \\
\hline Entrance pupil diameter & $31.0 \mathrm{~mm}$ \\
\hline Field of View & $\begin{array}{l}38.4 \mathrm{~km} \times 38.4 \mathrm{~km} \\
2.59^{\circ} \times 2.59^{\circ}\end{array}$ \\
\hline F/number & 1.6 \\
\hline Spectral range & {$[9.75 ; 11.25] \mu \mathrm{m}$} \\
\hline Pixel size & $34 \mu \mathrm{m}$ (binning of $2 \times 2$ native pixel a $17 \mu \mathrm{m})$ \\
\hline Nyquist frequency & $14.7 \mathrm{cycles} / \mathrm{mm}$ \\
\hline
\end{tabular}

The detector selected for the FEI is the ULIS1024-048 and it is shown in Figure 11 8. This is composed of an array of 1024 x $76817 \mu \mathrm{m}$ pitch microbolometer resistors made from amorphous Silicon connected to Si readout circuit (ROIC) and integrated into vacuum Kovar package. The micro-bolometer array will be operated at a controlled temperature around ambient $(295 \mathrm{~K} \pm 2.5 \mathrm{~K})$ by controlling the temperature of the detector housing. The detector housing will have a cone shape and will provide a uniform temperature background for the major part of the detector field of view. The front-end electronics will be located close to the detector on the other side of the optical bench. These electronics amplify and digitize the signal. The digitized raw signals are formatted in the ICU and handed over to the platform for transmission to ground. 


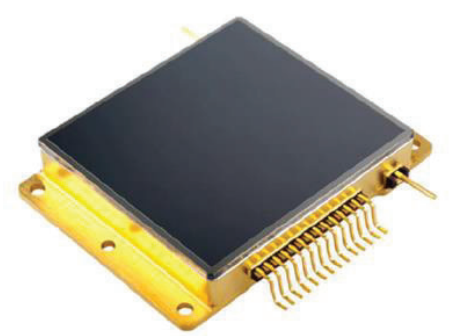

Figure 17 ULIS1024-048 $\mu$ bolometer detector package.

\subsection{Instrument Structure}

The instrument structure will consist of a monolithic lightened optical bench made in aluminum 6061, which carries the FSI subsystems and also the FEI instrument. The baseplate will be mounted with three bipods to the platform panel.

\subsection{Instrument Thermal Control}

The instrument shall be mounted to the platform panel on the $\mathrm{Z}_{-\mathrm{SC}}$ side as shown in Figure 18 . There it lies in the shadow of the platform with minimum temperature variations around the orbit. Using a two-stage insulation system (sunshield and instrument MLI), the instrument can be decoupled from its surroundings, reaching an undercooled temperature level with slight temporal and spatial temperature gradients. Using this initial cold state, the system can be reliably stabilized with PI controlled heaters located on the bottom side of the optical bench. The units with significant dissipative power possess their own thermal control system consisting radiators, heaters and PI controllers.

The thermal design will keep the instrument in the temperature regime around ambient $(295 \mathrm{~K} \pm 5 \mathrm{~K})$. This is close to the temperature of the calibration blackbody and of the detector.

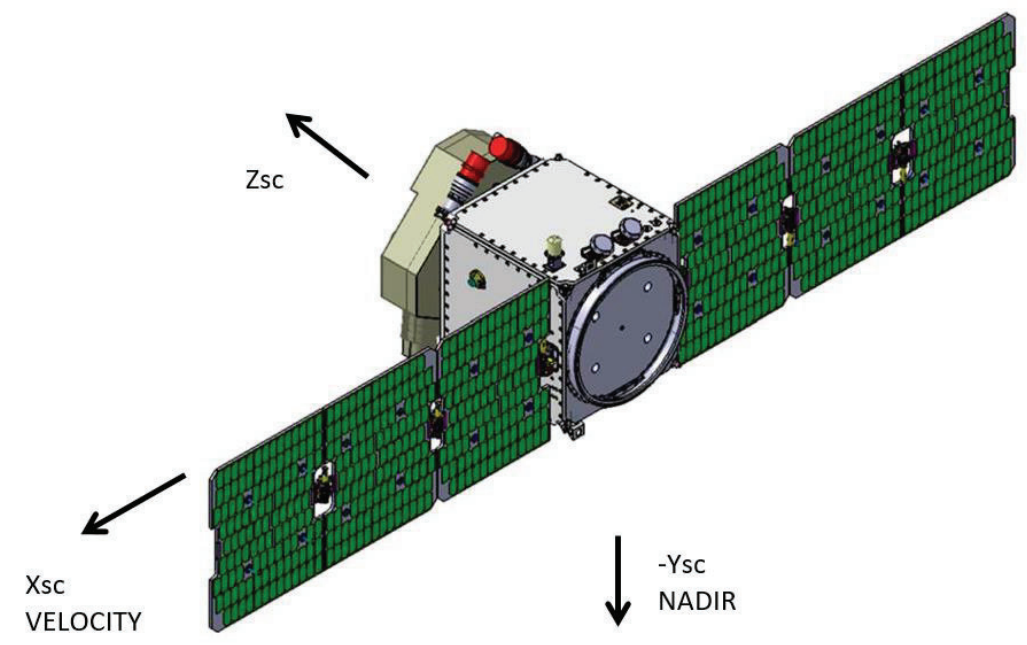

Figure 18 Mounting position of the instrument on the platform

\subsection{Instrument Modes, Instrument Operation and Synchronization}

Two observation modes are required:

- $\quad$ Earth observation mode to acquire TOA spectral radiances,

- Calibration mode.

With respect to synchronisation of the mechanisms, the reference is the fringe counter of the laser metrology system. 
There are two clocks relevant for the instrument:

- The absolute clock linked to the platform clock. The platform will provide the absolute clock as Central On Board Time (COBT) via the MilBus. Together with the Pulse Per Second (PPS) the ICU will synchronise to this absolute COBT.

- The relative clock, based on fringe counts of the laser interferometer. Relevant for the operation of the instrument is the relative clock, by counting the laser fringes.

The relative clock will deviate somewhat from the absolute clock, caused by interferometer speed fluctuations, by laser wavelength drift, fringe count losses etc. So once in a while the interferometer may be calibrated by determining the optical ZPD position (with help of the mechanical ZPD reference) and then symmetrical fringe counts from this position on. The mechanical zero reference gives an indication of the rotation position of the interferometer without processing the calibration signal.

Laser frequency drift: The spectral axis will rely on the frequency stability of the laser. The spectral position of significant absorption lines (e.g. CO2) can be checked periodically on ground versus nominal line position. If a given threshold deviation is exceeded a correction factor will be derived and uploaded, which takes the possible laser frequency shift into account. This could then be taken into account for the fringe counter.

Both interferometer position and laser frequency calibration do not require a special mode. The first can in principle be part of any blackbody calibration and the second is only ground processing. Between these calibrations the interferometer control electronics compares the relative clock with the absolute clock and corresponding speed adjustments will be commanded. This shall ensure that all scene acquisition requirements are within spec.

\subsection{Radiometric Calibration}

A two-point calibration scenario is proposed with cold space as zero reference and an on-board blackbody at or close to the maximum scene temperature of $300 \mathrm{~K}$. Both calibration sources will be acquired via a pointing mirror, which is also used to acquire Nadir position for Earth observations.

The instrument duty cycle is $100 \%$, which means there are no orbit positions dedicated for calibrations. Therefore, a natural assumption is a systematic $20 \%$ duty cycle for calibrations. The calibration cycles will be equidistant in time and not synchronised with the orbit duration, and therefore, no systematic loss of coverage will occur.

The cold space is a zero reference and does not contain spectral features. The blackbody shall have a broadband coating without high-resolution spectral features. Any remaining spectral features of the blackbody and optical elements are expected to be resolvable with a resolution of 3 to $4 \mathrm{~cm}^{-1}$ in calibration mode.

This allows significant reduction of the spectral sampling of the calibration measurements without compromising the radiometric accuracy. As a preliminary assumption a factor 10 reduction in sampling is proposed, i.e. $3.7 \mathrm{~cm}^{-1}$ instead of $0.37 \mathrm{~cm}^{-1}$. The blackbody Planck function is then still sampled with 405 samples in the wavenumber range of interest (100 $\mathrm{cm}^{-1}$ to $1600 \mathrm{~cm}^{-1}$ ). Any residual higher spectral resolution features will be regularly controlled with full spectral resolution of $0.36 \mathrm{~cm}^{-1}$ (see Section below).

The advantage of this approach is a significant improvement in SNR.

\section{Calibration Scenario}

The proposed calibration scenario assumes that 3 out of 15 science acquisitions being dedicated to calibrations, see below. This is in total $20 \%$ loss of observations. 


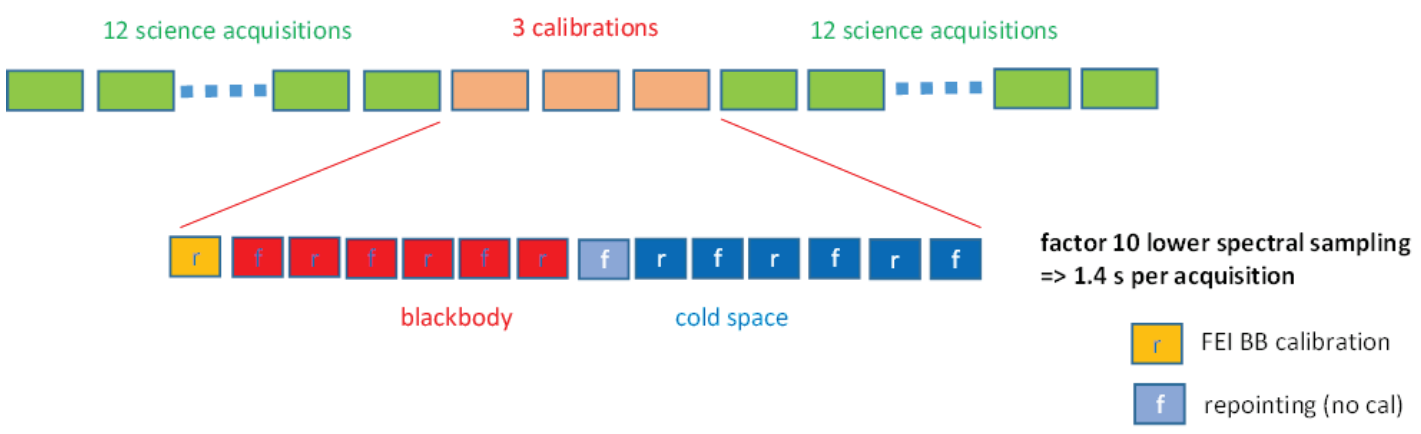

Figure 19 Proposed calibration strategy

Assuming $97.5 \mathrm{~km} \mathrm{SSD}$, the period between acquisitions is $14.8 \mathrm{~s}$. For interferometer turn-around and scan mirror repositioning $0.8 \mathrm{~s}$ are allocated. Then the useful interferogram acquisition time is $14.0 \mathrm{~s}$. Total time between two science acquisitions are $3 \times 14.8 \mathrm{~s}=44.4 \mathrm{~s}$.

The proposed timeline is shown in the Figure below. Assumed are 3 blackbody acquisitions and 3 cold space acquisitions for each of the two interferometer scan directions. Assuming $1.4 \mathrm{~s}$ per calibration IFG, the spectral sampling becomes 3.7 $\mathrm{cm}^{-1}$.

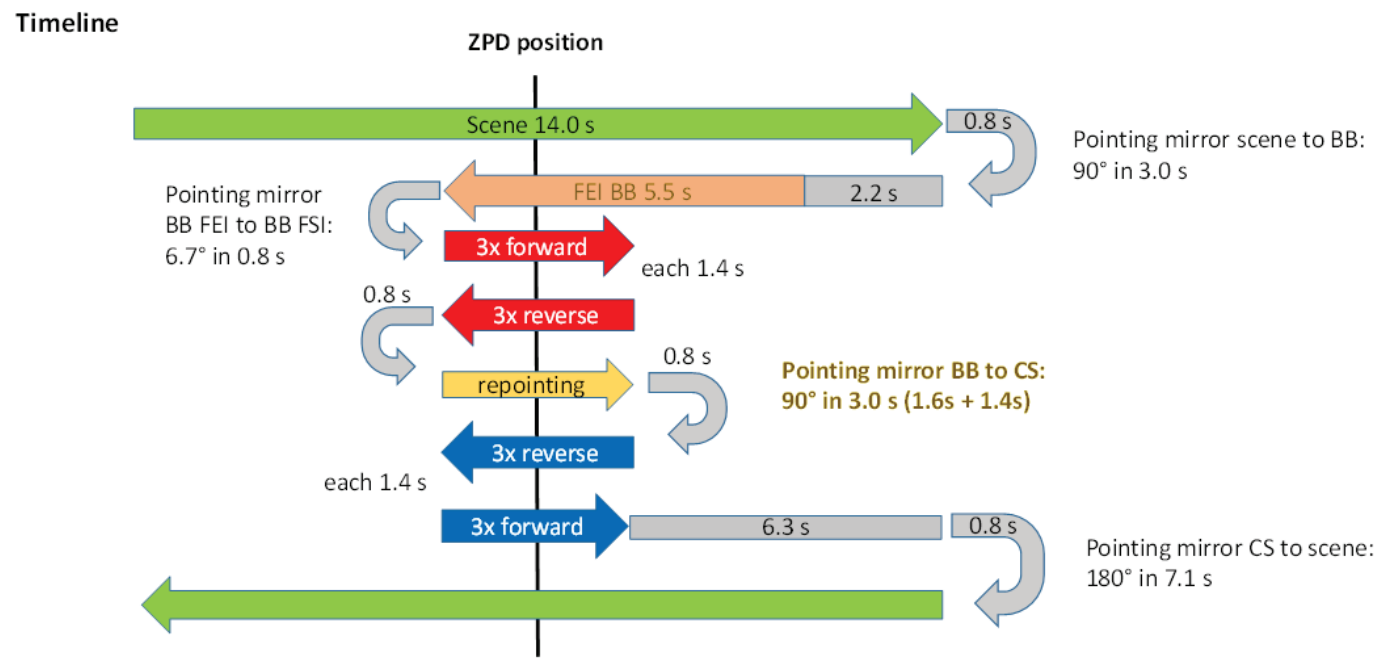

Figure 20 Proposed calibration timeline

The pointing mirror has to acquire large angles: the angular distance Nadir to blackbody is $90^{\circ}$ and blackbody to cold space $90^{\circ}$. Going back from cold space to the scene is $180^{\circ}$. The calibration timeline takes this into account and adds 2 interferometer sweeps for pointing mirror movement. The tolerable time for $90^{\circ}$ acquisition is then $3.0 \mathrm{~s}$ and $7.1 \mathrm{~s}$ are allocated for the $180^{\circ}$ angle including time for stabilization.

The total duration in the described calibration scenario is $14 \times 0.8 \mathrm{~s}+2 \times 6.3 \mathrm{~s}+14 \times 1.4 \mathrm{~s}=43.4 \mathrm{~s}$. This is $1.0 \mathrm{~s}$ less than allowed. The effective calibration time is then $19.55 \%$ and compliant to the maximum of $20 \%$.

\section{Check for Spectral Features}

The stability of instrument spectral features originating from e.g. the coatings can be checked infrequently with full spectral resolution. For example once per month for about one to two orbits.

For example 
- 400 measurement with $2.0 \mathrm{~cm}$ MPD taking $143.8 \mathrm{~min}$,

- 200 blackbody acquisitions (100 forward and 100 reverse),

- 200 cold space acquisitions (100 forward and 100 reverse).

This increases the effective calibration time by $0.33 \%$ to $19.88 \%$.

\section{FSI PERFORMANCE}

\subsection{Geometrical Performance}

The geometrical performance of the FSI instrument is mainly determined by the point spread function at the plane of the telescope's field stop. It is specified by the system integrated energy (SIE) and inhomogeneity of the system energy distribution function (SEDF). Even if the instrument images the scene on a single pixel detector, the shape of the SEDF understood as a relative system transmission dependent on the field point puts constraints on the image quality. This is in particular driving for the far infrared wavelengths above $20 \mu \mathrm{m}$, where the size of the Airy-disc becomes critically large due to diffraction. Furthermore, field dependent transmission effects at optical elements even after the field stop influence the SEDF homogeneities. In total the following effects contributes to the geometrical performance: aberration, diffraction, Coating transmission, in-orbit stability, micro-vibrations and stray light.

In the Figure 21 the calculated SEI and SEDF are depicted for the most critical wavelength of $100 \mu \mathrm{m}$. In this calculation based on an optical model only the aberrations, diffraction and coating effects are included. Micro-vibration and stray light are assessed separately and are considered negligible. The achieved values wrt. the requirements are listed in Table 5 .
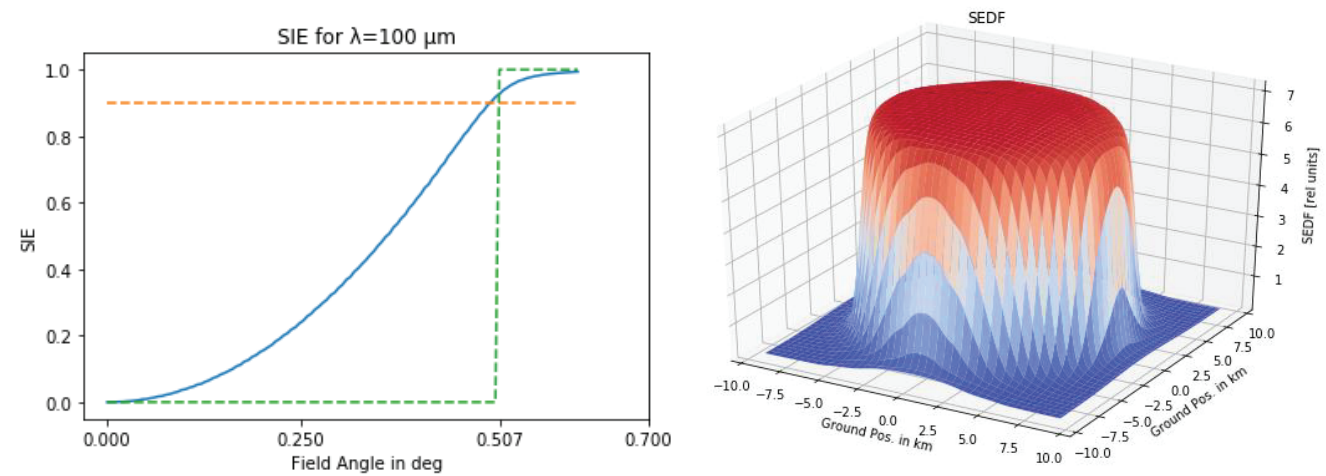

Figure 21 Geometrical performance at the most critical wavelength of $100 \mu \mathrm{m}$ given as SIE (left) and SEDF (right)

Table 5 Geometrical performance compliance status

\begin{tabular}{|l|l|l|}
\hline Parameter & Specification & Design \\
\hline SEI at $\mathbf{1 0 0 \mu \mathrm { m }}$ & $>90 \%$ & $>92 \% \quad \rightarrow$ compliant \\
\hline SEDF inhomogeneity at $\mathbf{1 0 0} \mu \mathrm{m}$ & $<15 \%$ & $<6.4 \% \rightarrow$ compliant \\
\hline
\end{tabular}

\subsection{FSI Spectral Performance}

The spectral performance is specified as Instrument Spectral Response Function (ISRF). Its deviation from a perfect Sincfunction is dominated by self-apodisation.

Below, on the left subplot is visible the self-apodisation envelope expected in the interferogram domain. The two central subplots show the normalized ILS (normalized such that the self-apodisation-free condition would reach 1) and unitary where each ILS is normalized to have a maximum at 1 (favourable to determine the FWHM for the spectral resolution). The subplot on the right shows the expected spectral resolution with and without the impact of the instrument radiometric response. There is a small margin to be compliant with the threshold spectral resolution of $0.5 \mathrm{~cm}^{-1}$. 

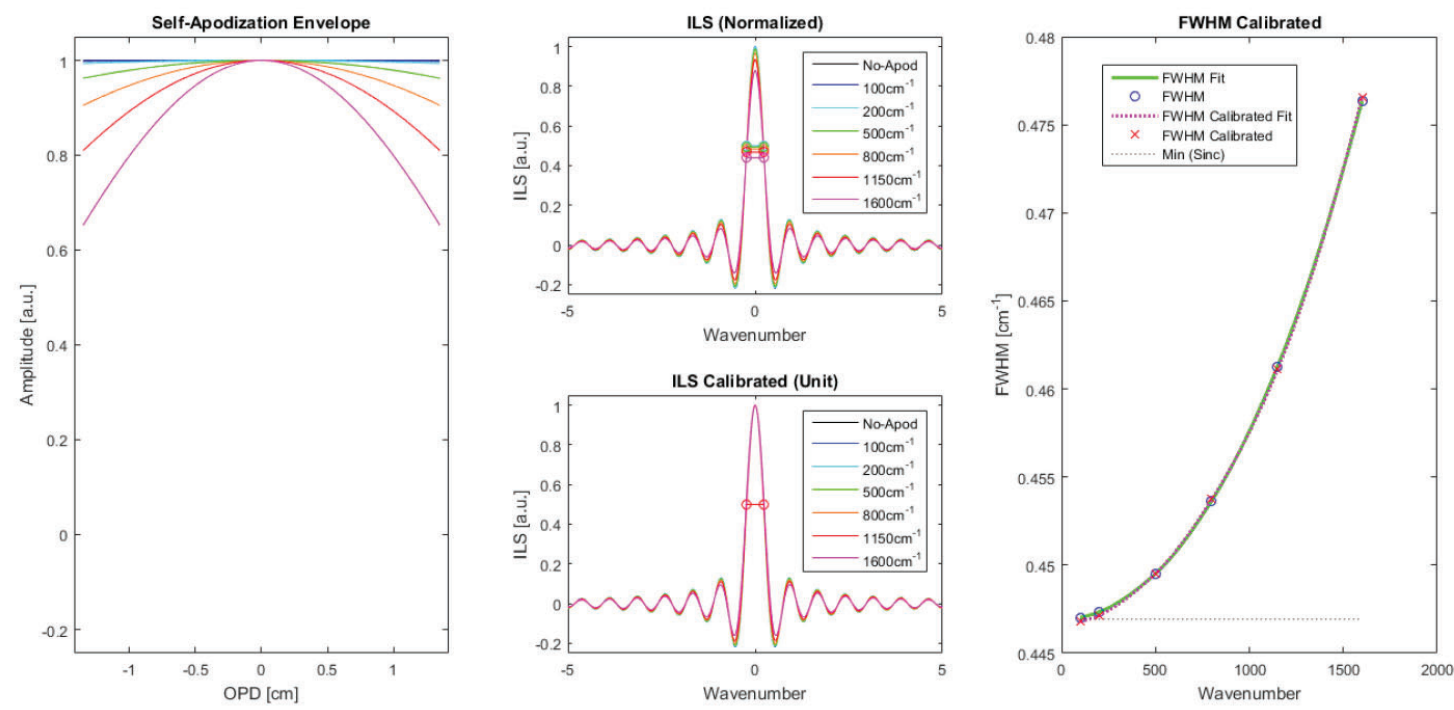

Figure 22 Results for the spectral performance

Table 6 Summary of spectral requirement performance

\begin{tabular}{|l|l|l|}
\hline Spectral parameter & \multicolumn{1}{|c|}{ Requirement } & \multicolumn{1}{|c|}{ Performance } \\
\hline Wavenumber range & 100 to $1600 \mathrm{~cm}^{-1}$ & 100 to $1600 \mathrm{~cm}^{-1} \rightarrow$ compliant \\
\hline Spectral resolution & Threshold $0.5 \mathrm{~cm}^{-1}\left(\mathrm{Goal} 0.36 \mathrm{~cm}^{-1}\right)$ & $<0.477 \mathrm{~cm}^{-1} \quad \rightarrow$ compliant to threshold \\
\hline Spectral accuracy & Threshold $2 \mathrm{ppm}(\mathrm{Goal} 1 \mathrm{ppm})$ & $<0.83 \mathrm{ppm} \quad \rightarrow$ compliant to goal \\
\hline ISRF asymmetry & Threshold $<3.34 \%($ Goal $<1.67 \%)$ & $<0.5 \%$ \\
\hline
\end{tabular}

\subsection{FSI Radiometric Performance}

The NESR is one of the key performances of the FSI instrument of FORUM. It was known from the beginning that the instrument is detector limited due to the necessity to use pyroelectric detectors to cover the desired spectral bandwidth. At PRR, the design was tuned to reach with margin the threshold requirement with the minimum guaranteed performance of DLATG detectors from Leonardo that have been flown in the past (TRL5). At SRR, the instrument is optimized and the detector performance is based on manufactured and tested batch after radiation aging (10 krad total dose).

The Table 7 presents the radiometric requirement performance.

Table 7 Summary of radiometric requirement performance

\begin{tabular}{|c|c|c|c|c|c|c|c|c|}
\hline Radiometric & \multicolumn{4}{|c|}{ Requirement } & \multicolumn{4}{|c|}{ Performance } \\
\hline \multirow{4}{*}{ NESR } & \multicolumn{2}{|c|}{ Wavenumber Range $\left[\mathrm{cm}^{-1}\right]$} & \multirow{2}{*}{\begin{tabular}{|c}
$\begin{array}{c}\text { Threshold } \\
{\left[\mathrm{mW} /\left(\mathrm{m}^{2} \mathrm{sr} \mathrm{cm}^{-1}\right)\right]}\end{array}$ \\
2
\end{tabular}} & \multirow{2}{*}{\begin{tabular}{|c|}
$\begin{array}{c}\text { Goal } \\
{\left[\mathrm{mW} /\left(\mathrm{m}^{2} \mathrm{sr} \mathrm{cm}^{-1}\right)\right]}\end{array}$ \\
1 \\
\end{tabular}} & \multicolumn{2}{|c|}{ Wavenumber Range $\left[\mathrm{cm}^{-1}\right]$} & \multicolumn{2}{|c|}{$\begin{array}{c}\text { Performance } \\
{\left[\mathrm{mW} /\left(\mathrm{m}^{2} \mathrm{sr} \mathrm{cm}^{-1}\right)\right]}\end{array}$} \\
\hline & 100 & 200 & & & 100 & 200 & 0.37 & $<$ Goal \\
\hline & 200 & 800 & 0.6 & 0.4 & 200 & 800 & 0.28 & $<$ Goal \\
\hline & 800 & 1600 & 2 & 1 & 800 & 1600 & 0.52 & $<$ Goal \\
\hline
\end{tabular}




\begin{tabular}{|c|c|c|c|c||c|c|c|c|}
\hline \multirow{4}{*}{$\begin{array}{c}\text { Absolute } \\
\text { Radiometric }\end{array}$} & \multicolumn{2}{|c|}{ Wavenumber Range $\left[\mathrm{cm}^{-1}\right]$} & Threshold $[\mathrm{K}]$ & Goal $[\mathrm{K}]$ & Wavenumber Range $\left[\mathrm{cm}^{-1}\right]$ & \multicolumn{2}{|c|}{ Performance $[\mathrm{K}]$} \\
\cline { 2 - 10 } & 100 & 200 & 1 & 1 & 100 & 200 & 0.191 & $<$ Goal \\
\cline { 2 - 10 } Accuracy & 200 & 300 & 0.2 & 0.25 & 200 & 300 & 0.064 & $<$ Goal \\
\cline { 2 - 10 } & 300 & 1100 & 0.1 & 0.25 & 300 & 1100 & 0.095 & $<$ Goal \\
\cline { 2 - 10 } & 1100 & 1300 & 0.2 & 0.25 & 1100 & 1300 & 0.192 & $<$ Goal \\
\cline { 2 - 9 } & 1300 & 1600 & 1 & 1 & 1300 & 1600 & 0.973 & $<$ Goal \\
\hline
\end{tabular}

The LEFT part of the following figure provides the expected instrument efficiency components and EoL detector sensitivity level D*. The predicted NESR performance is showed in the RIGHT part of the figure. The NESR is already compliant to the goal NESR with the balanced port detector and offers some margins with both detectors combined.
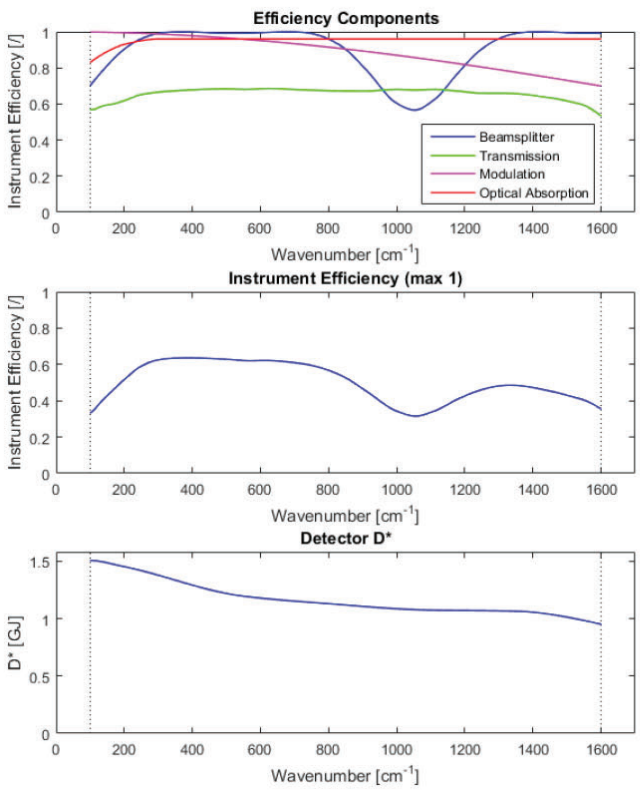

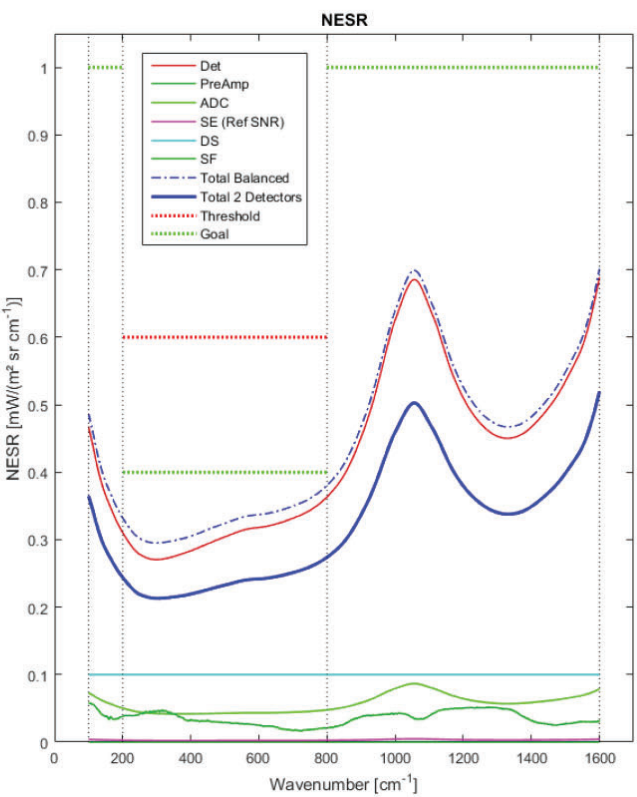

Figure 23(LEFT) Instrument input parameters and (RIGHT) NESR performance prediction

\section{CONCLUSION}

The design of the FORUM instrument as developed by the consortium lead by Thales Alenia Space and OHB System AG was presented. The FSI instrument is based on a compact double pendulum interferometer with the beam splitter as a key component implemented as CVD diamond windows providing an improved optical transmission over the specified spectral range from $6.25 \mu \mathrm{m}$ to $100 \mu \mathrm{m}$. Together with the black body performance and the detection chain based on pyroelectric detector the current instrument design fulfills all requirements, in particular the challenging spectral, radiometrical and geometrical specifications.

\section{ACKNOWLEDGEMENTS}

The authors acknowledge that this work is ESA funded through the Earth Explorer 9 program. 